TRANSACTIONS OF THE

AMERICAN MATHEMATICAL SOCIETY

Volume 184, October 1973

\title{
STABILITY OF FOLIATIONS
}

\author{
BY
}

\author{
HAROLD I. LEVINE( $\left.{ }^{1}\right)$ AND MICHAEL SHUB( ${ }^{2}$ )
}

\begin{abstract}
Let $X$ be a compact manifold and let $k$ be an integer. It is shown that the set of homeomorphism conjugacy classes of germs at $X$ of foliations of codimension $k$ and the set of homeomorphism conjugacy classes of (holonomy) representations of $\boldsymbol{n}_{1}(X)$ in the group of germs at 0 of 0 -fixed selfdiffeomorphisms of $\mathbf{R}^{k}$ are homeomorphic when given appropriate topologies. Stable foliation germs and stable holonomy representations correspond under this homeomorphism. It is shown that there are no stable foliation germs at a toral leaf if the dimension of the torus is greater than one.
\end{abstract}

0. Introduction. The qualitative theory of ordinary differential equations on compact manifolds without boundary may be considered a part of the theory of foliations, but the question of stability which has dominated so much of the recent work on vector fields and diffeomorphisms has been largely untouched for foliations. The relation between global and local perturbations is different for the two theories; whereas every local perturbation of a vector field extends to a global perturbation, the same is not true for foliations. This can be seen by comparing Hirsch's sufficient condition for global stability (quoted below) with our nonexistence theorem for local stable foliations at a toral leaf.

In trying to generalize certain stability theorems for vector fields one can assume the existence of a vector field tangent to the leaves which has a stabilizing effect on the foliation. For example.

Theorem (Hirsch). A foliation of a compact manifold which admits a normally byperbolic vector field is stable.

In the same spirit, another easy consequence of [H.P.S.] is the local theorem of persistence of a compact leaf:

Theorem. Let $X$ be a leaf of a foliation $\phi$. Suppose there is a neighborbood $U$ of $X$ and a vector field in $U$ tangent to $\phi$ and normally byperbolic at $X$. Then given a $C^{1}$-small perturbation $\tilde{\phi}$ of $\phi$ in $U$, there is an embedding $i: X \rightarrow U$, $C^{1}$-close to the inclusion of $X$ in $U$, such that $\tilde{i}(X)$ is a leaf of $\tilde{\phi}$.

Received by the editors August 9. 1972, and, in revised form, January 30, 1973. AMS (MOS) subject classifications (1970). Primary 57D30.

(1) This work was done while the author was partially supported by NSF GP28323.

(2) This work was done while the author was partially supported by NSF GP28375. 
On the other hand at a compact leaf $X$ of a foliation of codimension $k$ one has the holonomy representation. This is a representation, defined up to conjugation by elements of $D_{\text {iff }}\left(R^{k}, 0\right)$, of $\Pi_{1}(X)$ in Diff $\left(R^{k}, 0\right)$, the germs of local diffeomorphisms of $\mathbf{R}^{k}$ at the origin which leave the origin fixed. This is the generalization of the Poincaré transform. The bolonomy group of $X$ is the image of the holonomy representation; this too is only defined up to conjugacy.

Theorem (Reeb stability theorem). Let $X$ be a compact leaf of a differentiable foliation. If the bolonomy of $X$ is finite then $X$ bas a fundamental system of neighborboods each of which is a union of leaves.

That the stability in these two results arises because of completely different reasons is made clear by

Proposition. Let $X$ be a leaf of foliations of a compact manifold which admits a normally byperbolic vector field. Then $X$ admits a nonvanisbing vector field and if $X$ is compact $\Pi_{1}(X)$ is infinite.

Proof. Suppose the vector field tangent to $X$ has a singularity, the strong stable manifold of the singularity [H.P.S.] would contradict the completeness of the leaves. If $X$ is compact take a recurrent orbit, close it up and observe that the image of the homotopy class of that curve under the holonomy representation is hyperbolic and hence the homotopy class is of infinite order. We shall concern ourselves with local perturbations of foliations in a neighborhood of a compact leaf and stability questions arising from such perturbations. It is interesting that we can prove (see $\$ I I$ ) that a foliation is never locally stable in the neighborhood of a toral leaf $T^{k}$ for $k>1$, whereas many of the globally stable foliations of Hirsch have toral leaves. Thus it is clear that there are local perturbations that do not extend and that the global stability of a foliation is strongly affected by the integrability conditions.

In part I, the notions of stability of a compact leaf of a germ of a foliation are defined. The object of $\$ I$ is to identify the space $\mathcal{F}$ of homeomorphism equivalence classes of foliation germs in a neighborhood of a compact $n$-manifold $X$ embedded in $(n+k)$-dimensional manifolds, with a subspace $\left\{H^{1}\left(X, D_{i f f} R^{k}\right)\right\}$ of $H^{1}\left(X\right.$, Homeo $\left.\mathbf{R}^{k}\right)$. The subspace $\mathcal{F}_{X}$ of those foliation germs at $X$ having $X$ as a leaf is identified with the space $\left\{\operatorname{Rep}\left(\Pi_{1}(X), \operatorname{Diff}\left(\mathbf{R}^{k}, 0\right)\right)\right\}$ of homeomorphism conjugacy classes of holonomy representations.

This identification allows us to reduce the study of stability of foliations $\bmod X$ to the study of stability of holonomy representations.

In part II, this reduction is exploited in order to show that there are no stable foliation germs mod a toral leaf $T^{k}$ for $k>1$. 
While this paper only deals with the stability of foliations in the neighborhood of a given compact leaf, i.e. stability in $\mathcal{F}_{X}$, Hirsch [Hirsch ${ }_{2}$ ] has dealt with the persistence of the compact leaf under perturbations.

I. Foliation germs at a compact manifold $X$; the basic homeomorphism theorem.

1. Let $M$ be an $(n+k)$-dimensional manifold of class $C^{t}, t \geq 1$. Let $F_{k}(M)$ be the set of $C^{t}$-foliations of codimension $k$. We fix $t, k$, and $n$ and delete them where possible from the notation. In the obvious way $F(M)$ is a subset of the $C^{t-1}$-sections of $G_{n}(T M)$ over $M, \Gamma\left(G_{n}(T M)\right)$. Here $G_{n}(T M)$ is the bundle over $M$ whose fibre over $x \in M$ is the Grassmann manifold of $n$-dimensional subspaces of $(T M)_{x} \cdot$ On $\Gamma\left(G_{n}(T M)\right)$, we put the $C^{t-1}$-Whitney topology, and we take $F(M)$ with the induced topology. Since the inclusion, $F(M) \rightarrow \Gamma\left(G_{n}(T M)\right)$ involves taking one derivative, we call this topology on $F(M)$ the $C^{t}$-topology.

Now let $X$ be a compact $n$-dimensional manifold. Our object here is to study perturbations of foliations having $X$ as a leaf, in a neighborhood of the $X$-leaf. These perturbations may not be restricted to those that preserve $X$ as a leaf. Thus we want to look at foliations of $(n+k)$-dimensional manifolds in the neighborhood of an imbedded $X$ where we make no assumptions that $X$ is a leaf of the foliation. However since we are interested in foliations close to ones having $X$ as a leaf we require that the leaves of the foliation are locally graphs over $X$. For our purposes, therefore, it is no restriction to consider foliations of a neighborhood of the zero section of an $\mathbf{R}^{k}$-bundle over $X$, whose leaves project nonsingularly to $X$.

Let $\nu$ be an $\mathbf{R}^{k}$-bundle over $X$ with projection $\pi_{\nu}$ and zero section $z_{\nu}$. Let $U$ be an open neighborhood of $z_{\nu}(X)$, and let $F_{\nu}(U)$ be the set of those foliations $\sigma \in F(U)$ such that

$$
\left(\pi_{\nu}\right)_{*}(\sigma(u))=(T X)_{\nu}(u)
$$

We topologize $F_{\nu}(U)$ as a subset of $F(U)$, with the $C^{t}$-topology. We let $F_{\nu, X}(U)$ $C F_{\nu}(U)$ be the subspace of those foliations $\sigma$ such that $\sigma\left(z_{\nu}(x)\right)=\left(z_{\nu}\right)_{*}\left(T X_{x}\right)$. Let $F_{\nu}=\bigcup F_{\nu}(U)$ and $F_{\nu_{0} X}=\bigcup F_{\nu, X}(U)$, where the union is taken over all open neighborhoods $U$ of $z_{\nu}(X)$ and the $F_{\nu}(U)$ are disjoint for distinct $U$. Finally let $F=U F_{\nu}$ and $F_{X}=U_{F_{\nu} X}$ where this union is taken over all $\mathbf{R}^{k}$-bundles over $X$. Thus $F$ and $F_{X}$ are disjoint unions of topological spaces; they are given the obvious topologies.

For each $r, 0 \leq r \leq t$, we introduce an equivalence relation in $F$. Let $\sigma_{i} \epsilon$ $\Gamma\left(G_{n}\left(T U_{i}\right)\right)$ represent foliations in $F_{v_{i}} i=1$, 2. We say that $\sigma_{1}$ and $\sigma_{2}$ are $C^{r}$-equivalent at $X$ if there are open sets $V_{i}$ of $z_{\nu_{i}}(X)$ in $U_{i}$ and a $C^{r}$-diffeomorphism $b: V_{1} \rightarrow V_{2}$ such that 
(1)

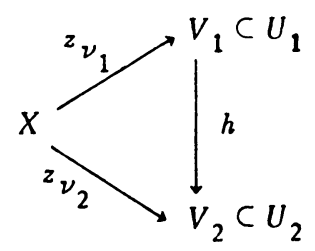

commutes.

(2) $b$ takes leaves to leaves.

Note that $F_{X}$ is a union of equivalence classes. For any $r, 0 \leq r \leq t$, we denote by $\mathcal{F}^{r}$ and $\mathcal{F}_{X}^{r}$ the quotients of $F$ and $F_{X}$ respectively by the $C^{r}$-equivalence relation and give each the quotient topology. Thus we have $\mathcal{F}_{X}$ a subspace of $\mathfrak{F}$.

If $\nu$ is an $\mathbf{R}^{k}$-bundle over $X$, let $\mathcal{F}_{\nu}$ and $\mathcal{F}_{\nu, X}$ be the images of $F_{\nu}$ and $F_{\nu_{0} X}$ in $\mathcal{F}_{r}^{r}$ and $\mathcal{F}_{X}^{r}$, respectively; $\mathcal{F}_{\nu}^{r}$ and $\mathcal{F}_{\nu, X}^{r}$ are open in $\mathcal{F}^{r}$ and $\mathcal{F}_{X}^{r}$, respectively.

Note. Hereafter, we will omit the superscript $r$ when $r=t$; that is, we will write $\mathcal{F}_{\text {for }} \mathcal{F}^{t}$ etc.

The set $\mathcal{F}_{\nu}$ is the set of germs at $z_{\nu}(X)$ of $C^{t}$-foliations, and the topology on $\mathcal{F}_{\nu}$ is defined by the $(t-1)_{\text {-jet at }} z_{\nu}(X)$ of the germ of the section into the Grassmannian which defines the foliation.

For any $r, 0 \leq r \leq t$, the forgetful map $\mathcal{F}_{X} \rightarrow \mathfrak{F}_{X}^{r}$ is continuous.

Definition. A foliation class $\sigma \in \mathcal{F}_{X}$ is $C^{r}$-stable if the forgetful map $\mathcal{F}_{X} \rightarrow$ $\mathcal{F}_{X}^{r}$ is constant on a neighborhood of $\sigma$.

Questions. (1) Is the $C^{r}$-stability of $\sigma$ equivalent to: the image of $\sigma$ in $\mathcal{F}_{X}^{r}$ is an isolated point?

(2) Is the forgetful map $\mathcal{F}_{X} \rightarrow \mathcal{F}_{X}^{r}$ open?

Definition. A foliation in $F_{X}$ is $C^{r}$-stable at $X$ if its image in $\mathcal{F}_{X}$ is r-stable.

Generally we shall be interested in $C^{0}$-stability, thus when we say that a foliation (or its class) is stable we will mean $C^{0}$-stable.

2. Let $\mathbf{H o m e o}^{r}\left(\mathbf{R}^{k}\right)$ be the set of germs of local $C^{r}$-diffeomorphism of $\mathbf{R}^{k}$, and let Homeo ${ }^{r}\left(\mathbf{R}^{k}, 0\right)$ be the group of those elements of $\mathrm{Homeo}^{r}\left(\mathbf{R}^{k}\right)$ with source and target equal to the origin. For the integer $t$ introduced in the preceding paragraph, we let $\operatorname{Homeo}^{t}\left(\mathbf{R}^{k}\right)=\operatorname{Diff}^{k}$ and $\operatorname{Homeo}^{t}\left(\mathbf{R}^{k}, 0\right)=\operatorname{Diff}\left(\mathbf{R}^{k}, 0\right)$. We topologize Diff $R^{k}$ by means of the $t$-jets at the source; that is we give it the weakest topology making the map continuous.

$$
\text { Diff } \mathbf{R}^{k} \rightarrow J^{t}\left(\mathbf{R}^{k}, \mathbf{R}^{k}\right): f \rightarrow J^{t} f(x)=\left(x, f(x), j^{t} f(x)\right),
$$

where $f$ is a germ at $x$. Call this topology on Diff $\mathbf{R}^{k}$ and the induced topology on the subset Diff $\left(\mathbf{R}^{k}, 0\right)$, the $C^{t}$-topology.

In distinction to the $C^{t}$-topology, we have the usual sheaf topology on Homeo $^{r}\left(\mathbf{R}^{k}\right)$, for any $r$. A basic open set in this topology is $(f, U)$ the set of germs of $f$ at all points of $U$ where $U$ is an open set in $\mathbf{R}^{k}$ and $f$ is a 
$C^{r}$-diffeomorphism of $U$ into $\mathbf{R}^{k}$. Note that in this topology $\mathbf{D}_{\text {iff }}\left(\mathbf{R}^{k}, 0\right)$ is discrete. We use the sheaf topology on $D_{i f f} R^{k}$ and $D_{i f f}\left(R^{k}, 0\right)$ to define the sets $H^{1}\left(X, D_{\text {iff }} \mathbf{R}^{k}\right)$ and $H^{1}\left(X, \operatorname{Diff}\left(\mathbf{R}^{k}, 0\right)\right)$. The inclusion of $\mathbf{D i f f}^{k}$ in $\mathbf{H o m e o}^{r}\left(\mathbf{R}^{k}\right)$ for $r \leq t$ induces a map from $H^{1}\left(X, \mathrm{Diff}^{k}\right)$ into $H^{1}\left(X, \mathrm{Homeo}^{r} \mathbf{R}^{k}\right)$. We denote the image of this map by $\left\{H^{1}\left(X, D_{\text {iff }} \mathbf{R}^{k}\right)\right\}^{r}$. Similarly, $\left\{H^{1}\left(X, \operatorname{Diff}\left(\mathbf{R}^{k}, 0\right)\right)\right\}^{r}$ is the image of $H^{1}\left(X, \operatorname{Diff}\left(\mathbf{R}^{k}, 0\right)\right)$ in $H^{1}\left(X, \operatorname{Homeo}^{r}\left(\mathbf{R}^{k}, 0\right)\right)$.

To topologize $H^{1}\left(X, \operatorname{Diff} \mathbf{R}^{k}\right)$ and $H^{1}\left(X, \operatorname{Diff}\left(\mathbf{R}^{k}, 0\right)\right)$, it suffices to topologize, in a consistent way, the set of 1 -cochains $C^{1}\left(\mathfrak{U}, D_{i f f} R^{k}\right)$ for $\mathfrak{U}$ a finite open cover of $X$. But $C^{1}\left(\mathfrak{l}, D_{i f f} R^{k}\right)$ is just the product over all pairs $U, V, \epsilon \mathfrak{U}$ such that $U \cap V \neq \varnothing$ of the sets $C\left(U \cap V\right.$, Diff $\left.R^{k}\right)$ of continuous maps of $U \cap V$ into Diff $\mathbf{R}^{k}$ (with the sheaf topology). We give $C\left(U \cap V\right.$, Diff $\left.\mathbf{R}^{k}\right)$ the topology of uniform convergence using the $C^{t}$-topology on Diff $\mathbf{R}^{k}$. Notice that the analogously defined topology on $C^{1}\left(\mathfrak{U}, \operatorname{Diff}\left(\mathbf{R}^{k}, 0\right)\right)$ identifies $C^{1}\left(\mathfrak{U}, \operatorname{Diff}\left(\mathbf{R}^{k}, 0\right)\right)$ as a subspace of $\left(\operatorname{Diff}\left(R^{k}, 0\right)\right)^{q}$ where $q$ is the number of pairs $U, V \in \mathfrak{U}$ with $U \cap V \neq \varnothing$.

By a result of Haefliger $\left(\left[\mathrm{H}_{1}\right.\right.$, p. 303], $\left[\mathrm{H}_{2}\right.$, p. 382] or $\left[\mathrm{H}_{3}\right.$, p. 188]) there are $1: 1$ correspondences

$$
\mathcal{F} \stackrel{h}{\rightarrow} H^{1}\left(X, D_{i f f} R^{k}\right) \text { and } \mathscr{F}_{X} \stackrel{h}{\rightarrow} H^{1}\left(X, D_{\text {iff }}\left(\mathbf{R}^{k}, 0\right)\right)
$$

which commute with the inclusions $\mathcal{F}_{X} \rightarrow \mathcal{F}$ and $H^{1}\left(X, \operatorname{Diff}\left(R^{k}, 0\right)\right) \rightarrow$ $H^{1}\left(X\right.$, Diff $\left.\mathbf{R}^{k}\right)$. It is obvious that for each $r$ this correspondence $b$ projects to a $1: 1$ correspondence $b^{r}$ in the commutative diagram:

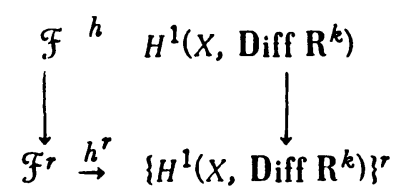

Since the topologies on $\mathcal{F}^{r}$ and $\left\{H^{\mathrm{I}}\left(X, \mathrm{Diff}^{k}\right)\right\}$ are quotient topologies of the two vertical maps, to prove $b^{r}$ is a homeomorphism it suffices to prove that $b$ is a homeomorphism. The proof of this is carried out in $\$ 4$.

For each $r, 0 \leq r \leq t$, there is another space homeomorphic to $\mathfrak{F}_{X}^{r}$, namely the space of $C^{r}$-conjugacy classes of representations of $\Pi_{1}(X)$ into $\operatorname{Diff}\left(R^{k}, 0\right)$. The correspondence associates to each $C^{r}$-foliation class the $C^{r}$-class of its holonomy representation.

Let $\operatorname{Rep}\left(\Pi_{1}(X), \operatorname{Diff}\left(R^{k}, 0\right)\right)$ be the set of representations of $\Pi_{1}^{*}(X)$ into the group $\operatorname{Diff}\left(\mathbf{R}^{k}, 0\right)$, and let $\left\{\operatorname{Rep}\left(\Pi_{1}(X), \operatorname{Diff}\left(\mathbf{R}^{k}, 0\right)\right)\right\}$ be the set of equivalence classes of such representations, where two representations are equivalent if they are conjugate by an element of $\operatorname{Homeo}^{r}\left(\mathbf{R}^{k}, 0\right)$, the group of germs of local homeomorphisms of $\mathbf{R}^{k}$ at 0 . Where no confusion is likely to arise we will write simply $\operatorname{Rep}$ and $\{\operatorname{Rep}\}^{r}$. 
We topologize Rep as follows: Since $\Pi_{1}(X)$ is finitely presented we can choose a finite set of generators $\gamma_{1}, \cdots, \gamma_{r}$ and map

$$
\begin{aligned}
\gamma: \operatorname{Rep} & \longrightarrow \operatorname{Diff}\left(\mathbf{R}^{k}, 0\right)^{r} \\
\rho & \longrightarrow\left(\rho\left(\gamma_{1}\right), \ldots, \rho\left(y_{r}\right)\right)
\end{aligned}
$$

and put the $\gamma$-induced topology on Rep. This topology is independent of the choice of generators. In fact if $\eta_{1}, \cdots \eta_{s}$ is another set of generators, then there are words $w_{i}\left(\gamma_{1}, \cdots, \gamma_{r}\right)=\eta_{i}$ and $v_{j}\left(\eta_{1}, \cdots, \eta_{s}\right)=\gamma_{j}$ which define continuous maps $w$ and $v$, making the following diagram commute:

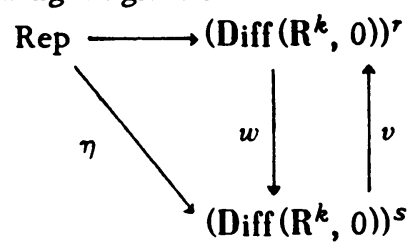

Thus in the $\gamma$-induced topology, $\eta=w \circ \gamma$ is continuous so the $\gamma$-induced topology contains the $\eta$-induced topology.

We give $\left\{\operatorname{Rep}\left(\Pi_{1}(X), \operatorname{Diff}\left(\mathbf{R}^{k}, 0\right)\right)\right\}^{r}$ the quotient topology.

Definition. A representation $\rho \in \operatorname{Rep}\left(\Pi_{1}(X), \operatorname{Diff}\left(R^{k}, 0\right)\right)$ is $C^{r}$-stable if the forgetful map

$$
\operatorname{Rep}\left(\Pi_{1}(X), \operatorname{Diff}\left(\mathbf{R}^{k}, 0\right)\right) \rightarrow\left\{\operatorname{Rep}\left(\Pi_{1}(X), \operatorname{Diff}\left(\mathbf{R}^{k}, 0\right)\right)\right\}^{r}
$$

is constant on a neighborhood of $\rho$.

In the next section we establish a homeomorphism between

$$
\left\{H^{1}\left(X, \operatorname{Diff}\left(\mathbf{R}^{k}, 0\right)\right)\right\}^{r} \text { and }\left\{\operatorname{Rep}\left(\Pi_{1}(X), \operatorname{Diff}\left(\mathbf{R}^{k}, 0\right)\right)\right\}^{r}
$$

for $0 \leq r \leq t$.

Our results can be summarized:

Theorem 1. (a) The map $b: \mathcal{F} \rightarrow H^{1}\left(X\right.$, Diff $\left.\mathbf{R}^{k}\right)$ is a bomeomorpbism wbicb projects to a bomeomorphism $b^{r}$ sucb that the following diagram commutes:

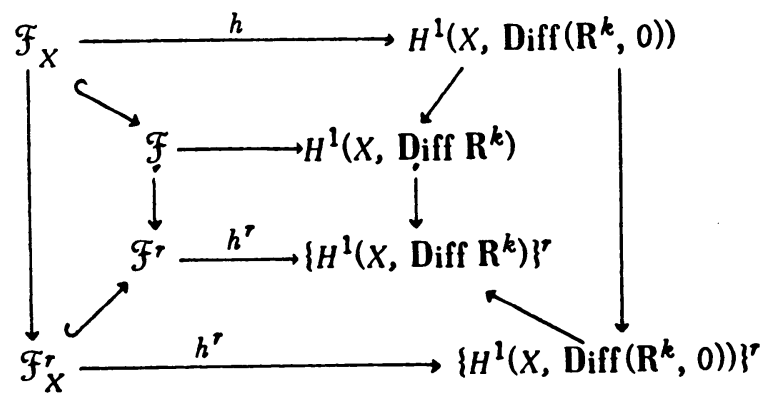


(b) $\left\{H^{1}\left(X, \operatorname{Diff}\left(\mathbf{R}^{k}, 0\right)\right)\right\}^{r}$ and $\left\{\operatorname{Rep}\left(\Pi_{1}(X), \operatorname{Diff}\left(\mathbf{R}^{k}, 0\right)\right)\right\}^{r}$ are bomeomorpbic and the bomeomorphisms commute with the forgetful maps. In particular the follow. ing diagram commutes:

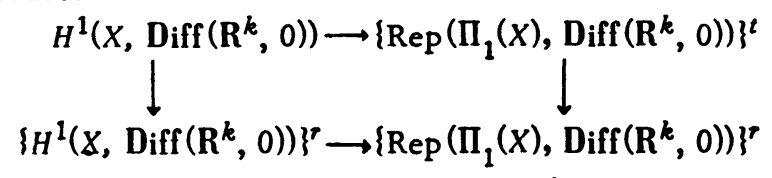

(In both parts $0 \leq r \leq t$ and the topologies are the $C^{t}$-topologies for $t \geq 1.0$ )

From this theorem we see that the study of stability of foliations at $X$ is equivalent to the study of stability of representations of $\Pi_{1}(X)$ in $\operatorname{Diff}\left(R^{k}, 0\right)$. More generally, if $G$ is any subgroup of $\operatorname{Diff}\left(\mathbf{R}^{k}, 0\right)$ with the inherited $C^{t}$-topology and $\mathcal{F}_{X}^{r}, G$ is the image under $\left(b^{r}\right)^{-1}$ of $\left\{H^{1}(X, G)\right\}$, then

Corollary. There are bomeomorphisms $k^{r}(0 \leq r \leq t)$ such that the following diagram commutes:

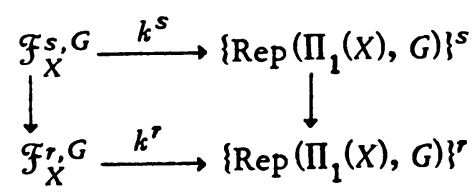

for $0 \leq r \leq s \leq t$ : the vertical maps are the forgetful projections.

Here $\left\{\operatorname{Rep}\left(\Pi_{1}(X), G\right)\right\}^{r}$ is the image under the forgetful projection of $\operatorname{Rep}\left(\Pi_{1}(X), G\right)$ in $\left\{\operatorname{Rep}\left(\Pi_{1}(X), \operatorname{Diff}\left(\mathbf{R}^{k}, 0\right)\right)\right\}$. The proof of the corollary is merely the proof that $\left\{H^{1}(X, G)\right\}^{r}$ and $\left\{\operatorname{Rep}\left(\Pi_{1}(X), G\right)\right\}^{r}$ are naturally (with respect to forgetfulness) homeomorphic. This is proved in the next paragraph.

3. In this paragraph we construct a homeomorphism between $\left\{H^{1}(X, G)\right\}^{r}$ and $\left\{\operatorname{Rep}\left(\Pi_{1}(X), G\right)\right\}^{r}$ for any $r, 0 \leq r \leq t$, and $G$, any subgroup of $\operatorname{Diff}\left(\mathbf{R}^{k}, 0\right)$. Since $r$ is fixed in this paragraph we will suppress it.

Let $\mathfrak{U}=\left\{U_{1}, \cdots, U_{u}\right\}$ be a finite open cover by coordinate balls such that if $U_{i} \cap U_{j}=U_{i j} \neq \varnothing$ then $U_{i} \cup U_{j}$ is simply connected and if $U_{i} \cap U_{j} \cap U_{k}=$ $U_{i j k} \neq \varnothing$ then $U_{i} \cup U_{j} \cup U_{k}$ is simply connected. We will show that $\left\{H^{1}(\mathfrak{a}, G)\right\}$ and $\left\{\operatorname{Rep}\left(\Pi_{1}(X), G\right)\right\}$ are homeomorphic. From the construction given below, it it clear that if $\mathfrak{B}$ is any covering of $X$ of the same type that refines $\mathfrak{U}$ then the homeomorphism of $\left\{H^{1}(\Re, G)\right\}$ and $\left\{\operatorname{Rep}\left(\Pi_{1}(X), G\right)\right\}$ can be defined so that

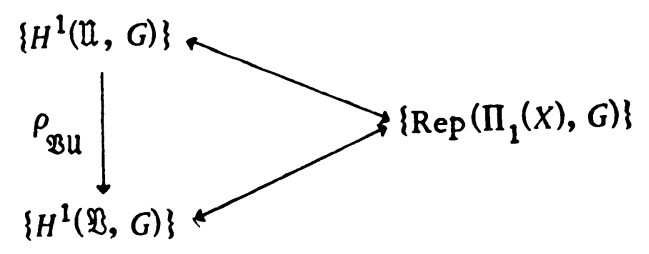


commutes, where $\rho_{\mathcal{B}}$ is the refinement map. Thus we will have defined a homeomorphism of $\left\{H^{1}(X, G)\right\}$ and $\left\{\operatorname{Rep}\left(\Pi_{1}(X), G\right)\right\}$.

Let $Z^{1}(\mathfrak{U}, G)=Z^{1}$ be the set of 1 -cocycles based on the covering $\mathfrak{U}$. Let $q$ be the number of pairs $(i, j)$ with $1 \leq i, j \leq u$ such that $U_{i j} \neq \varnothing$. Then as mentioned in $\$ 2, Z^{1}$ is just a subset of $G^{q}$. Let $\lambda$ be the inclusion of $Z^{1}$ in $G^{q}$.

For each $U_{i} \in \mathfrak{U}$, choose a point $x_{i} \in U_{i}, i=1, \cdots, u$; we construct a set of $q$ generators for $\Pi_{1}\left(X, x_{1}\right)$. For each $U_{i j} \neq \varnothing, i<j$, choose an arc $D_{i j}$ in $U_{i} \cup U_{j}$ joining $x_{i}$ to $x_{j}$. We will denote by $D_{j i}, D_{i j}^{-1}$, and $D_{i i}$ is the constantly $x_{i}$ path. For each $x_{i}$, choose a path $C_{i}$ from $x_{1}$ to $x_{i}$ having the form $D_{1 j_{2}} D_{j_{2} j_{3}} \cdots D_{j_{l} i}$. For $C_{1}$ we take the constant path. Let $\gamma_{i j}$ be the homotopy class of $C_{i} D_{i j} C_{j}^{-1}$. Since any closed path at $x_{1}$ is homotopic to one of the form $D_{1 j_{2}} D_{j_{2} j_{3}} \cdots D_{j_{r} 1}$, the set $\left\{\gamma_{i j}\right\}$ obviously generates $\Pi_{1}\left(x_{1} x_{1}\right)$. Using this set of generators define the map $\gamma$ from $\operatorname{Rep}=\operatorname{Rep}\left(\Pi_{1}\left(X, x_{1}\right), G\right)$ into $G^{q}$. By the definition of the topology in $\operatorname{Rep}$ and $Z^{1}$ we have two continuous inclusions

$$
\gamma: \operatorname{Rep} \rightarrow G^{q} \leftarrow Z^{1}: \lambda
$$

The image of $\gamma$ is contained in the image of $\lambda$. To see this we need merely show that if $U_{i j k} \neq \varnothing$ that $\gamma(\rho)_{i j}{ }^{\circ} \gamma(\rho)_{j k}=\gamma(\rho)_{i k}$ but

$$
\begin{aligned}
\gamma(\rho)_{i j} \circ \gamma(\rho)_{j k} & =\rho\left(\gamma_{i j}\right) \circ \rho\left(\gamma_{j k}\right)=\rho\left(\gamma_{i j} \gamma_{j k}\right)=\left(\left[C_{i} D_{i j} C_{j}^{-1}\right]\left[C_{j} D_{j k} C_{k}^{-1}\right]\right) \\
& =\rho\left(\left[C_{i} D_{i j} D_{j k} C_{k}^{-1}\right]\right)=\rho\left(\left[C_{i} D_{i k} C_{k}^{-1}\right]\right)=\rho\left(\gamma_{i k}\right)=\gamma(\rho)_{i k}
\end{aligned}
$$

Here we have used the simple connectivity of $U_{i} \cup U_{j} \cup U_{k}, D_{i j} D_{j k} D_{k i} \simeq 1$. Thus we have a $1: 1$ continuous map $\phi: \operatorname{Rep} \rightarrow Z^{1}$ defined by $\lambda \circ \phi=\gamma$. To construct a map in the other direction we define a map $r$ from $G^{q}$ to itself so that the image of $\tau \circ \lambda$ is contained in the image of $\gamma_{\bullet}$

We have chosen as representative of $\gamma_{i j}$ a word in the paths $\left\{D_{k l}\right\}$ which begins with $D_{1}$. and ends with $D_{\cdot 1^{\circ}}$. Call this word $r_{i j}(D) ; r_{i j}$ is a word in $q$ symbols. Thus we may define $r: G^{q} \rightarrow G^{q}$ by $(r(g))_{i j}=r_{i j}(g)$ for $g \in G^{q}$. This map is obviously continuous. To show that the image of $r \circ \lambda$ is in the image of $\gamma$, let $g$ be in the image of $\lambda$. Define $\rho\left(\gamma_{i j}\right)=\tau_{i j}(g)$. We must show that $\rho$ thus defined on the generators of $\Pi_{1}\left(X, x_{1}\right)$ extends to a representation. This means that if $w$ is any word in $q$ symbols and if $w\left(\gamma_{i j}\right)=1$ then $w\left(\tau_{i j}(g)\right)=1$. Or if $w\left(\tau_{i j}(D)\right)$ is null homotopic then $w\left(\tau_{i j}(g)\right)=1$. But the word $w\left(\tau_{i j}(D)\right)$ is null homotopic iff it can be reduced to the trivial word $D_{11}$ by a sequence of substitutions of the form

(1) $D_{k l} D_{l j}$ replacing $D_{k j}$, if $U_{j k l} \neq \varnothing$,

(2) $D_{k j}$ replacing $D_{k l} D_{l j}$, if $U_{j k l} \neq \varnothing$.

However if $g$ is in the image of $\lambda$, for each nonempty $U_{j k l}$ we have $g_{k l} g_{l j}=g_{k j}$. Thus the image of $r \circ \lambda$ is in the image of $\gamma$. We define a continuous map 
$\theta: Z^{1} \rightarrow \operatorname{Rep}$ by $\tau \circ \lambda=\gamma \circ \theta$. Notice that $\tau$ is defined so that $\tau \circ \gamma=\gamma$. For let $\rho \in \operatorname{Rep}$ and suppose $\gamma_{i j}=\left[D_{1 k_{1}} \cdots D_{i j} \cdots D_{l_{1}}\right]$. Then

$$
\begin{aligned}
& r_{i j}(\gamma(\rho))=\rho\left(\gamma_{1 k_{1}}\right) \cdots \rho\left(\gamma_{i j}\right) \cdots \rho\left(\gamma_{l_{1} 1}\right) \\
& \quad=\rho\left(\gamma_{1 k_{1}} \cdots \gamma_{i j} \cdots \gamma_{l_{1}}\right)=\rho\left[D_{1 k_{1}} D_{k_{1} k_{2}} \cdots D_{i j} \cdots D_{l_{1} 1}\right]=\rho\left(\gamma_{i j}\right)=(\gamma(\rho))_{i j}
\end{aligned}
$$

Thus we have constructed continuous maps making the diagrams commute:
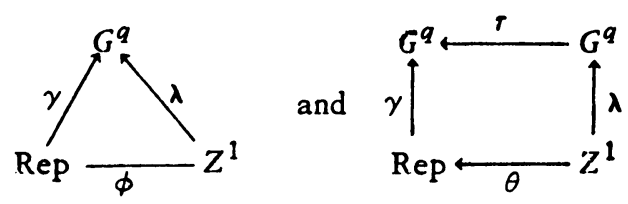

Thus $\tau \circ \gamma=\tau \circ \lambda \circ \phi=\gamma \circ \theta \circ \phi$. Since $\tau \circ \gamma=\gamma$ we have $\gamma=\gamma \circ \theta \circ \phi$ so $\theta \circ \phi=1_{\text {Rep }}$. So we have $\theta$ is surjective. Suppose $\rho$ and $\rho^{1}$ are conjugate representations. Then there is a homeomorphism germ $b$ such that

$$
\rho^{1}\left(\gamma_{i j}\right)=b \rho\left(\gamma_{i j}\right) b^{-1}, \quad \gamma\left(\rho^{1}\right)_{i j}=b\left(\gamma(\rho)_{i j}\right) b^{-1} .
$$

Thus $\phi(\rho)$ and $\phi\left(\rho^{1}\right)$ define the same element in $\left\{H^{1}\right\}^{r}$. Call the resulting continuous map, $[\phi]:\{\operatorname{Rep}\} \rightarrow\left\{H^{1}\right\}$. Also if $g$ and $g^{1}$ in $Z^{1}$ define the same element of $\left\{H^{1}\right\}$, that is, if $g_{i j}^{1}=b_{i} g_{i j} b_{i}^{-1}$ for $b_{i}, b_{j}$ in $\operatorname{Homeo}^{r}\left(\mathbf{R}^{k}, 0\right)$, then $\theta\left(g^{1}\right)$ is the $b_{1}$-conjugate of $\theta(\mathrm{g})$. Thus $\theta$ also defines a continuous map [ $\left.\theta\right]$ :

$\left\{H^{1}\right\} r \rightarrow\{\operatorname{Rep}\}$. We already know that $[\theta] \circ[\phi]=1_{\{\text {Rep }\} r^{\circ}}$ To see that $[\phi] \circ[\theta]=$ $1_{\left\{H_{1}\right\}}$ note that

$$
\begin{aligned}
(\phi \circ \theta)(g)_{i j} & =\theta(g)\left(\gamma_{i j}\right)=(\gamma(\theta(g)))_{i j}=(\gamma(\lambda(g)))_{i j} \\
& =\tau_{i j}(g)=\left(g_{i j_{1}} \cdots g_{j_{l}}\right) g_{i j}\left(g_{j k_{r}} \cdots g_{k_{1} l}\right)=b_{i} g_{i j} b_{j}^{-1}
\end{aligned}
$$

where $C_{i}=D_{1 j_{1}} \cdots D_{j_{l} i}$ and $C_{j}=D_{1 k_{1}} \cdots D_{k_{r} j^{*}}$

This completes the proof of the fact that

Proposition 1. For any $r, 0 \leq r \leq t$, and any subgroup $G$ of $\operatorname{Diff}\left(R^{k}, 0\right)$, if $X$ is any compact manifold then $\left\{\operatorname{Rep}\left(\Pi_{1}(X), G\right)\right\}^{r}$ and $\left\{H^{1}(X, G)\right\}$ are bomeomorphic. These homeomorphisms commute with the forgetful projections.

4. In this paragraph we prove

Proposition 2. The 1:1 correspondence $b$ given by Haefliger (see $\$ 2$ ) is a bomeomorphism making the following diagram commute.

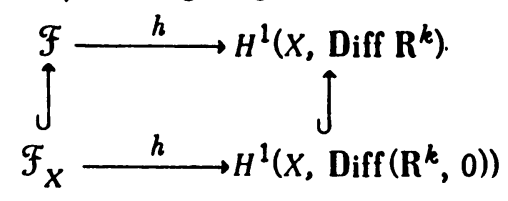


To show that $b$ is a homeomorphism, we restrict our attention to the open sets $\mathcal{F}_{\nu}$ and $\mathfrak{F}_{\nu, X}$ for $\nu$ an $\mathbf{R}^{k}$-bundle over $X$. We describe $\mathcal{F}_{\nu}$ and $\mathcal{F}_{\nu, X}$ in terms of coordinate-foliation germs at $z_{\nu}(X)$ (defined below) by means of distinguished functions $\left(\right.$ see $\left.\left[\mathrm{H}_{2}\right]\right)$. Since $\nu$ will be fixed for this paragraph we let $E$ be its total space, $\pi$ its projection, and $z$ its zero section.

Definition. A coordinate foliation germ at $z(X)$ consists of a pair $(\mathbb{U}, f)$ where $\mathfrak{U}$ is an open cover of $X$ and $f=\left\{f_{U} \mid U \in \mathfrak{U}\right\}$, where $f_{U}$ is a germ at $z(U)$ of a diffeomorphism of a neighborhood of $z(U)$ in $E \mid U$ into $U \times \mathbf{R}^{k}$ such that

(1) the following diagram commutes,

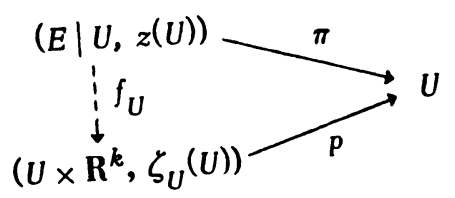

where $\zeta_{U}=f_{U} \circ z \mid U$ and $p$ is the projection on the first factor. The Mather broken arrow will be used to denote germs.

(2) If $U$ and $U^{\prime} \in \mathfrak{U}$ have nonempty intersection then there is a continuous function from $U \cap U^{\prime}$ into Diff $R^{k}$ (with the sheaf topology), $g_{U U^{\prime}}$, such that

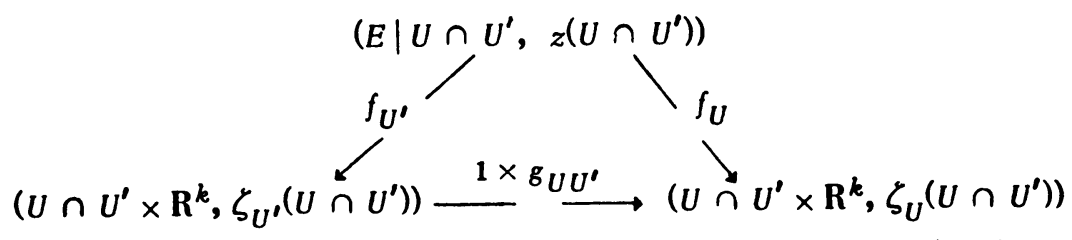
commutes, where if $\left(u, v^{\prime}\right)=\zeta_{U^{\prime}}(u)$ and $(u, v)=\zeta_{U}(u)$, then $g_{U U^{\prime}}(u, \cdot)$ is a germ of a local $C^{t}$-diffeomorphism of $\mathbf{R}^{k}$ with source $v^{\prime}$ and target $v$. Call $\{/ U\}$, the coordinate germs and $\left\{g_{U U^{\prime}}\right\}$, the transition germs of the coordinate foliation germ.

For a fixed covering $\mathfrak{U}$ of $X$, let $C_{\nu}(\mathfrak{U})$ be the space of coordinate foliation germs defined in terms of $\mathfrak{U}$. We topologize $C_{\nu}(\mathfrak{U})$ by declaring two elements $f$ and $f^{\prime}$ to be close if the ir $t$-jets; $\left(J^{t} f_{U}\right) \mid z(U)$ and $\left(J^{t} f_{U}^{\prime}\right) \mid z(U)$ are uniformly close. An element of $C_{\nu}(\mathfrak{l})$ determines an element of $\mathfrak{F}_{\nu}$, that is, there is an obvious map of $C_{\nu}(\mathfrak{U})$ into $\mathcal{F}_{\nu^{*}}$ Call the image of $C_{\nu}(\mathfrak{l})$ in $\mathcal{F}_{\nu}, \mathcal{F}_{\nu}(\mathfrak{I})$.

The collection of $C_{\nu}(\mathfrak{l})$ for all open covers $\mathfrak{U}$ is a directed system as is the collection of all $\mathfrak{F}_{\nu}(\mathfrak{U})$. The direct limit of $\left\{\mathfrak{F}_{\nu}(\mathfrak{U})\right\}$ is $\mathfrak{F}_{\nu}$. Replacing Diff $\mathbf{R}^{k}$ by $\operatorname{Diff}\left(R^{k}, 0\right)$ in the above definition, we can define $C_{\nu, x}(\mathfrak{l})$, and $\mathcal{F}_{\nu, x}(\mathfrak{l})$; again $\mathcal{F}_{\nu, X}$ is the direct limit of $\mathcal{F}_{\nu, X}(\mathfrak{U})$. The topologies on $\mathcal{F}_{\nu}(\mathfrak{U})$ and $\mathcal{F}_{\nu, X}(\mathfrak{U})$ are the quotient topologies of those of $C_{\nu}(\mathfrak{l})$ and $C_{\nu_{0}} X^{(\mathfrak{l}) \text {. }}$

Roughly speaking, $\operatorname{dir} \lim \left(C_{\nu, x}(\mathfrak{U})\right)$ is the set of microbundles with constant transition functions at $z(X)$ in $E$. An analogous imprecise identification could be made of $\operatorname{dir} \lim C_{\nu}(\mathfrak{l})$ if we had available a notion of microbundle without a zero section. 
The mapping $b: \mathcal{F} \rightarrow H^{1}\left(X\right.$, Diff $\left.\mathbf{R}^{k}\right)$ can be easily described on $\mathcal{F}_{\nu}(\mathfrak{M})$. Take a foliation which is the image of $f \in C_{\nu}(\mathfrak{I})$, the $b$-image of this foliation is the equivalence class of the 1-cocycle in $Z^{1}\left(\mathfrak{U}, D_{\text {iff }} R^{k}\right)$ given by $g=\left\{g_{U U^{\prime}} \mid U, U^{\prime} \in \mathfrak{U}\right\}$, the transition germs, where $f_{U}{ }^{\circ} f_{U}^{-1}=1 \times g_{U} U^{\prime}$. We determine the images under $b$ of $\mathcal{F}_{\nu}$ in $H^{1}\left(X, D_{\text {iff }} \mathbf{R}^{k}\right)$ and of $\mathcal{F}_{\nu}(\mathfrak{l})$ in $H^{1}\left(\mathfrak{U}, D_{\text {iff }} \mathbf{R}^{k}\right)$.

Consider the map $j:$ Diff $\left(\mathbf{R}^{k}\right) \rightarrow \mathrm{GL}(k, \mathbf{R}): \phi \rightarrow \phi^{\prime}(x)$ where $\phi$ is a germ of a diffeomorphism of $\mathbf{R}^{k}$ with source $x$, and $\phi^{\prime}(x)$ is the Jacobian of $\phi$ at $x$. This is a groupoid morphism and induces a map $j: H^{1}\left(X, D_{i f f} \mathbf{R}^{k}\right) \rightarrow H^{1}(X, \mathrm{GL}(k, \mathbf{R}))$. In the usual way, by means of transition functions, we consider $\nu \in H^{1}(X, \operatorname{GL}(k, R))$ and let $H_{\nu}^{1}\left(X, \operatorname{Diff} \mathbf{R}^{k}\right)$ be the preimage of $\nu$ under $j$, and let $H_{\nu}^{1}\left(X, \operatorname{Diff}\left(\mathbf{R}^{k}, 0\right)\right)$ be the $j$ preimage of $\nu$ in $H_{\nu}^{1}\left(X, \operatorname{Diff}\left(\mathbf{R}^{k}, 0\right)\right)$.

To complete the proof of Proposition 2 we prove

Lemma. (1) The b-image of $\mathcal{F}_{\nu}$ is contained in $H_{\nu}^{1}\left(X, D_{\text {iff }} \mathbf{R}^{k}\right)$.

(2) $H_{\nu}^{1}\left(X, D_{\text {iff }} R^{k}\right)$ is open in $H^{1}\left(X, D_{\text {iff }} \mathbf{R}^{k}\right)$.

(3) $b: \mathcal{F}_{\nu} \rightarrow H_{\nu}^{1}\left(X\right.$, Diff $\left.\mathbf{R}^{k}\right)$ is a bomeomorphism.

Proof. For (1) it suffices to prove that the $b$-image of $\mathcal{F}_{\nu}(\mathfrak{l})$ is contained in $H_{\nu}^{1}\left(\mathfrak{M}\right.$, Diff $\left.\mathbf{R}^{k}\right)$ for any open cover $\mathfrak{U}$ of $X$, by coordinate balls. Thus we look at the map $b$ cn the "coordinate" level: $b: C_{\nu}(\mathfrak{I}) \rightarrow Z^{1}(\mathfrak{l})$, taking a set $/$ of coordinate germs to the set $g$ of transition germs. In particular suppose $\mathfrak{U}=$ $\left\{U_{i}\right\}$, let $f_{i}=f U_{i} ; g_{i j}=g_{U_{i} U_{j}} ; E_{i}=E \mid U_{i}$ etc. Thus $b\left(\left\{f_{i}\right\}\right)=\left\{g_{i j}\right\}$ where $1 \times g_{i j}=f_{i} \circ f-1$. Suppose the local trivializations of $E$ are given relative to $\mathfrak{U}$ by $F_{i}: E_{i} \rightarrow U_{i} \times \mathbf{R}^{k}$. Thus the cocycle representing $\nu \cdot$ in $H^{1}(\mathfrak{l}, \mathrm{GL}(k, \mathbf{R}))$ is given by $\left\{G_{i j}\right\}$ where $1 \times G_{i j}=F_{i} \circ F_{j}^{-1}$, where $G_{i j}: U_{i j} \rightarrow \mathrm{GL}(k, \mathrm{R})$. Define $1 \times \lambda_{i}$ a germ at $U_{i} \times 0$ of a diffeomorphism of $U_{i} \times R^{k}$ by the commutativity of

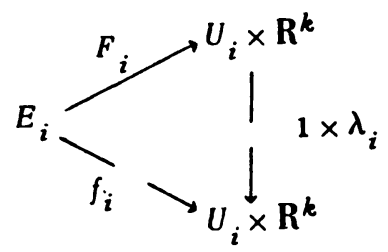

Thus we have the germ equation at $U_{i j} \times 0 \subset U_{i j} \times R^{k}$,

$$
\lambda_{j} \circ\left(1 \times G_{j i}\right)=g_{j i} \circ\left(1 \times \lambda_{i}\right) .
$$

Letting $D$ denote differentiation in the $\mathbf{R}^{k}$-direction, we obtain

$$
\left.\left(D \lambda_{j}\right)\right|_{U_{i j} \times 0} \cdot G_{j i}=\left.\left(D g_{j i}\right) \circ\left(1 \times\left.\lambda_{i}\right|_{U_{i j} \times 0}\right) \cdot D \lambda_{i}\right|_{U_{i j} \times 0}
$$

which gives us the equivalence of the $j$-image of the class of $\left\{g_{i j}\right\}$ with $\nu$. 
To prove (2) it suffices to show that $H^{1}(X, \mathrm{GL}(k, \mathbf{R}))$ is discrete, for which it suffices to show that for any open cover $\mathfrak{U}$ of $X, H^{1}(\mathfrak{U}, \mathrm{GL}(k, \mathbf{R}))$ is discrete. Thus we must show that if $g$ and $\tilde{g}$ are in $Z^{1}(\mathfrak{U}, G L(k, R))$ and sufficiently close, there exist continuous maps $\lambda_{U}: U \rightarrow \mathrm{GL}(k, \mathbf{R})$ such that $g_{U} U^{\prime}(x) \circ \lambda_{U^{\prime}}(x)=\lambda_{U}(x) \circ$ $\tilde{g}_{U U^{\prime}}(x)$, for all $U, U^{\prime} \in \mathfrak{U}$ and $x \in U \cap U^{\prime}$. The construction of such a 0 -chain $\lambda$ is a special case of the proof of (3).

To prove that $b: \mathcal{F}_{\nu} \rightarrow H_{\nu}^{1}\left(X\right.$, Diff $\left.\mathbf{R}^{k}\right)$ is a homeomorphism we note first that by (1) of this lemma and the above mentioned result of Haefliger that this restricted $b$ is $1: 1$ and onto.

Note. In the remainder of this paragraph we omit $D_{i f f} R^{k}$ and $X$ from the notation for $H_{\nu}^{1}, Z_{\nu}^{1}$, etc.

For each open cover $\mathfrak{U}$ of $X$, we have the commutative diagram

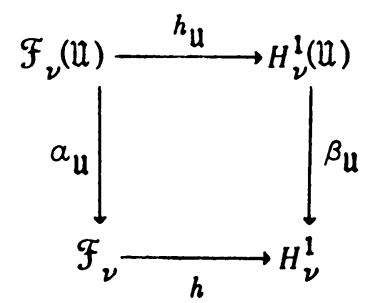

where $\alpha_{\mathfrak{u}}$ and $\beta_{\mathfrak{u}}$ are continuous. Thus the continuity of $b$ is a consequence of the continuity of $b_{\mathfrak{u}}$ which in turn is a consequence of the continuity of $C_{\nu}(\mathfrak{U}) \rightarrow Z_{\nu}^{1}(\mathfrak{H})$ which takes a set of coordinate germs into the corresponding transition germ cocycle. This last map is obviously continuous.

We prove that $b^{-1}$ is continuous by showing that for each open cover $\mathfrak{U}$ the map $b^{-1} \circ \beta_{\mathcal{U}}$ is continuous.

Since the projection $Z_{\nu}^{1}(\mathfrak{U}) \rightarrow H_{\nu}^{1}(\mathfrak{U})$ is open we need merely prove the continuity of

$$
Z_{\nu}^{1}(\mathfrak{l}) \rightarrow H_{\nu}^{1}(\mathfrak{l}) \stackrel{\beta_{\mathfrak{u}}}{\longrightarrow} H_{\nu}^{1} \stackrel{h^{-1}}{\longrightarrow} \mathcal{F}_{\nu}
$$

Remark. 1. It is sufficient to restrict attention to covers of $X$ by open coordinate balls.

Definition. Let $a:$ Diff $\mathbf{R}^{k} \rightarrow \mathrm{R}^{k}$ and $b:$ Diff $\mathbf{R}^{k} \rightarrow \mathrm{R}^{k}$ be the source and target maps. We say that a cocycle $g \in Z^{1}\left(\mathfrak{u}, D_{\text {iff }} R^{k}\right)$ is $C^{t}$ if

(i) $a\left(g_{i j}\right): U_{i j} \rightarrow \mathrm{R}^{k}$ is $C^{t}$ and

(ii) the germ of the map at the graph of $a\left(g_{i j}\right): \gamma_{i j}: U_{i j} \times \mathbf{R}^{k}-\rightarrow \mathbf{R}^{k}$, where $\gamma_{i j}\left(x,,^{\cdot}\right)$ is the germ $g_{i j}(x)$, is the germ of a $C^{t}$ map.

Obviously if $g$ is a $C^{t}$-cocycle, $b\left(g_{i j}\right)$ are also all $C^{t}$-maps.

2. Since $b: \mathcal{F}_{\nu} \rightarrow H_{\nu}^{1}$ is surjective, it is no restriction to compute $H_{\nu}^{1}$ using only $C^{t}$-cocycles. For the rest of this paragraph $Z_{\nu}^{1}(\mathfrak{U})$ means the set of $C^{t}$-cocycles. 
Definition. If $\mathfrak{U}$ is any open-ball covering of $X$, a proper refinement $\mathfrak{B}$ of $\mathfrak{U}$ is a refinement by open balls such that the cover, $\bar{B}$, the set of closures of the balls in $\mathfrak{B}$, also refines $\mathfrak{U}$.

The continuity of $b^{-1}$ is a consequence of the following:

Lemma. Let $\mathfrak{U}$ be a cover of $X$ by coordinate balls. Let $g \in Z_{\nu}^{1}(\mathfrak{U})$. Then there is a proper refinement $\mathfrak{B}$ of $\mathfrak{U}$ and a neigbborbood $\vartheta$ of $g$ and a continuous map

$$
\eta: \mathcal{O} \rightarrow C_{\nu}(\Re)
$$

sucb that the following diagram commutes:

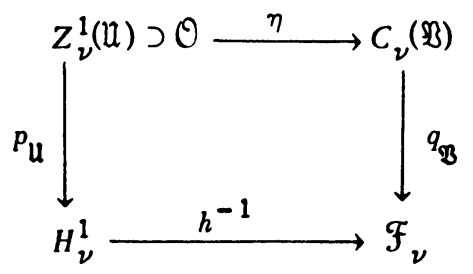

Here $p_{\mathrm{U}}$ and $q_{\mathfrak{B}}$ are the obvious continuous projections.

Proof. The refinement $B$ is any one such that $b^{-1} p_{\mathfrak{U}}(g) \in$ image of $q_{\mathfrak{g}}$. It is no restriction to assume that $\mathfrak{n}=\left\{U_{1}, \cdots, U_{u}\right\}$ and $\mathfrak{b}=\left\{v_{1}, \cdots, v_{u}\right\}$ and $v_{i} \subset \bar{V}_{i} \subset U_{i}$ for all $i$. Just take a refinement $\mathfrak{U}^{\prime}$ that has that property, construct the map for the pair of coverings $\mathfrak{U}^{\prime}$ and $\mathfrak{B}$ and compose it with the refining map, $Z_{\nu}^{1}(\mathfrak{U}) \rightarrow Z_{\nu}^{1}\left(\mathfrak{U}^{\prime}\right)$. Similarly we can assume that there is an $f \in C_{\nu}(\mathfrak{U})$ with $b q_{\mathfrak{u}}(f)=p_{\mathfrak{u}}(g)$. In fact we may assume that if $f=\left\{f_{i^{\prime}}, i=1, \ldots, u\right\}$ and $g=\left\{g_{i j}\right\}$ that

$$
f_{i} \circ f_{j}^{-1}=1 \times g_{i j}: U_{i j} \times \mathbf{R}^{k} \longrightarrow U_{i j} \times \mathbf{R}^{k}
$$

as germs with source $f_{j}\left(z\left(U_{i j}\right)\right)=$ graph of $a\left(g_{i j}\right)$ and target $f_{i}\left(z\left(U_{i j}\right)\right)=$ graph of $a\left(g_{j i}\right)$. Let $a_{i j}=a\left(g_{i j}\right): U_{i j} \rightarrow \mathbf{R}^{k}$. The scheme of the proof is as follows. For $\tilde{\boldsymbol{g}}$ close enough to $\boldsymbol{g}$, we construct a set of submersion germs:

$$
\lambda_{j}: U_{j} \times \mathbf{R}^{k}-\longrightarrow \mathbf{R}^{k} \text { with source } \bigcup_{i \neq j}\left(\text { graph } a_{i j}\right) \cup\left(U_{j}-\bigcup_{i \neq i} U_{i j}\right) \times \mathbf{R}^{k}
$$

where if we set $\tilde{f}_{j}=\left(1 \times \lambda_{j}\right) \circ f_{j}$, then

$$
\tilde{f}_{i} \circ \tilde{f}_{j}^{-1}=1 \times \tilde{g}_{i j}: V_{i j} \times \mathbf{R}^{k} \longrightarrow V_{i j} \times \mathbf{R}^{k}
$$

as germs at the graph of $a\left(\tilde{g}_{i j} \mid V_{i j}\right)$. Notice that there is no a priori contradiction here since if $U_{i j k} \neq \varnothing$ then $g_{i j}{ }^{\circ} g_{j k}=g_{i k}$ on $U_{i j k}$ so $a_{i j}=a_{i k}$ there. Thus $a\left(\lambda_{j}\right)$ is well defined on $\bigcup_{i \neq j} U_{i}$. The continuous dependence of $\lambda$ on $g, \tilde{g}$ and a fixed finite collection of partitions of unity is given explicitly below. The measure 
of proximity of $\tilde{g}$ to $g$ that defines the neighborhood $\theta$ is given by the conditions that the constructed $\lambda_{j}$ are all submersion germs. The fact that $\tilde{f}_{i} \circ \tilde{f}_{j}^{-1}=1 \times \tilde{g}_{i j}$ on the graph of $a\left(\tilde{g}_{i j} \mid V_{i j}\right)$ gives $b \circ q_{\mathfrak{B}}(\tilde{f})=b q_{\mathfrak{B}} \eta(\tilde{g})=p_{\mathfrak{U}}(\tilde{g})$, the commutativity of the diagram of the lemma.

Let $\tilde{g} \in Z^{1}(\mathfrak{l})$. Let $a\left(g_{i j}\right)=a_{i j}$ and $a\left(\tilde{g}_{i j}\right)=\tilde{a}_{i j}$; and let the graph of $a_{i j}=\widetilde{\Gamma}_{i j}$. We proceed to define the $\lambda$. As we go along we find the conditions on $\tilde{\boldsymbol{g}}$ which define $\mathcal{O}$, the domain of $\eta$. We define $\lambda$ in two steps. First for each $j=1, \cdots, u$, we define a submersion $\tau_{j}$ so that

$$
E_{j} \stackrel{f_{j}}{\longrightarrow} U_{j} \times \mathbf{R}^{k} \stackrel{1 \times r_{j}}{\longrightarrow} U_{j} \times \mathbf{R}^{k}
$$

the target, $b\left(1 \times r_{j} \circ f_{j}\right)$ agrees (on a slightly smaller cover) with the sources $a\left(1 \times \tilde{g}_{i j}\right)$. Thus at least as far as their sources and targets are concerned $\tilde{\boldsymbol{g}}_{i j}$ and $\left(1 \times r_{i}\right) \circ f_{j}^{-1} \circ\left(1 \times r_{j}\right)^{-1}$ agree. In the second step the diffeomorphisms are made to match up.

Let $\mathfrak{U}^{1}$ be a proper refinement of $\mathfrak{U}$ so that $\overline{\mathfrak{B}} \subset \mathfrak{U}^{1} \subset \overline{\mathfrak{U}}^{1} \subset \mathfrak{U}$ by which we mean $\mathfrak{U}^{1}=\left\{U_{1}^{1}, \cdots, U_{n}^{1}\right\}$ with

$$
\bar{V}_{i} \subset U_{i}^{1} \subset \bar{U}_{i}^{1} \subset U_{i} \text { for } i=1, \cdots, u \text {. }
$$

Let $j \in\{1, \cdots, u\}$; we define $\tau_{j}$. Let $\left\{\phi_{1}, \cdots, \phi_{u}\right\}$ be a partition of unity for $U_{j}$ subordinate to cover $\left\{U_{i j}, i \neq j, U_{j}-U_{i \neq j} \bar{U}_{i j}^{1}\right\}$ where the support of $\phi_{i}$ is contained in $U_{i j}$ and the support of $\phi_{j}$ is contained in $U_{j}-U_{i \neq j} \bar{U}_{i j}^{1}$. Define $\tau_{j}: U_{j} \times \mathbf{R}^{k} \rightarrow \mathbf{R}^{k}$ by:

$$
r_{j}(x, t)=t+\sum_{i \neq j} \phi_{i}(x)\left(\tilde{a}_{i j}(x)-a_{i j}(x)\right) .
$$

Since $f_{j}\left(z\left(U_{i j}\right)\right)=\Gamma_{i j}=\left\{\left(x, a_{i j}(x)\right) \mid x \in U_{i j}\right\}$ we see that for $x \in U_{i j}^{1}$ :

$$
\left(1 \times \tau_{j}\right) \circ f_{j}\left(f_{j}^{-1}\left(x, a_{i j}(x)\right)\right)=\left(x, a_{i j}(x)+\sum_{k \neq j} \phi_{k}(x)\left(\tilde{a}_{k j}(x)-a_{k j}(x)\right)\right) .
$$

For $x \in U_{i k j}, \tilde{a}_{k j}(x)=\tilde{a}_{i j}(x)$ and $a_{k j}(x)=a_{i j}(x)$. Thus

$$
\left(1 \times \tau_{j} \circ f_{j}\right)\left(z\left(U_{i j}\right)\right)=\widetilde{\Gamma}_{i j} \mid U_{i j}^{1}=\left\{\left(x, \tilde{a}_{i j}(x)\right) \mid x \in U_{i j}^{1}\right\} .
$$

Let $1 \times \tau_{j} \circ f_{j}=f_{j}^{1}, f_{i}^{1} \circ\left(f_{j}^{1}\right)^{-1}=g_{i j}^{1}, a_{i j}^{1}=a\left(g_{i j}^{1}\right)$ and $\Gamma_{i j}^{1}=$ graph of $a_{i j}^{1}$. We have $a\left(g_{i j}^{1}\right)=a\left(\tilde{g}_{i j}\right)$ on $U_{i j}^{p}$. We construct $\lambda_{j}, j=1, \cdots, u$, by induction on $j$ so that $\left(1 \times \lambda_{i}\right) \circ f_{i}^{1} \circ f_{j}^{1-1^{\prime}} \circ\left(1 \times \lambda_{j}\right)^{-1}=1 \times \tilde{g}_{i j}$ as germs of diffeomorphisms on $\widetilde{\Gamma}_{i j} \mid V_{i j}$. Let $\lambda_{1}: U_{1}^{1} \times \mathbf{R}^{k} \rightarrow \mathbf{R}^{k}$ be a projection on the second factor. Suppose we have completed $(p-1)$-steps of our induction. That is, we have an open cover $\mathfrak{H}^{p-1}$ such that $\bar{B} \subset \mathfrak{U}^{p-1}$ and a collection of submersion germs 


$$
\lambda_{j}: U_{j}^{p-1} \times \mathrm{R}^{k}-\rightarrow \mathrm{R}^{k} \text { with source } f_{j}^{1}\left(z\left(U_{j}^{p-1}\right)\right)
$$

$j=1, \cdots, p-1$, such that if $f_{j}^{p-1}=\left(1 \times \lambda_{j}\right) \circ f_{j}^{1}, j=1, \cdots, p-1, f_{j}^{p-1}=f_{j}^{1}$, $j \geq p$, and $1 \times g_{j k}^{p-1}=f_{j}^{p-1} \circ\left(f_{k}^{p-1}\right)^{-1}$, then

$$
g_{j k}^{p-1}=\tilde{g}_{j k} \text { on } U_{j k}^{p-1}, \text { for } 1 \leq j, k \leq p-1 .
$$

We still have $a\left(g_{j k}^{p-1}\right)=a\left(\tilde{g}_{j k}\right)$ on $U_{j k}^{p-1}$ for all $j, k_{0}$. Choose a proper refinement $\mathfrak{U}^{p}$ of $\mathfrak{U}^{p-1}$ such that $\overline{\mathfrak{B}} \subset \mathfrak{U}^{p} \subset \overline{\mathfrak{U}}^{p} \subset \mathfrak{U}^{p-1}$. To simplify the notation for this inductive step let $\left(e_{1}, \cdots, e_{u}\right)=\left(f_{1}^{p-1}, \ldots, f_{u}^{p-1}\right)$ and $e_{i} \circ e_{j}^{-1}=b_{i j}=g_{i j}^{p-1}$, and let $\mathfrak{U}^{p-1}=\mathfrak{B}^{p}$ and $\mathfrak{U}^{p}=\mathfrak{B}^{\prime}$. By our construction so far $b_{i j}=\tilde{g}_{i j} \mid W_{i j}, i \leq j \leq$ $p-1$, and on $W_{i j k}, b_{i j} \circ \tilde{g}_{j k}$ make sense for all $i, j, k$ (since we adjusted all the sources to be right in the last step). Let $\left\{\phi_{i}\right\}$ be a partition of unity for $W_{p}$ subordinate to the covering $\left\{W_{j ;}, j<p ; W_{p}-\bigcup_{j<p} \bar{W}_{j p}^{\prime}\right\}$, where the support of $\phi_{j}$ is contained in $W_{j p^{\prime}} j<p$, and $\phi_{p}=1-\Sigma_{j<p} \phi_{j} \cdot$

Define $\lambda_{p}$ by

$$
\left.\lambda_{p}(x)(t)=\sum_{j=1}^{p-1} \phi_{j}(x) \tilde{g}_{p j}(x) \circ h_{j p}(x)(t)\right)+\phi_{p}(x) t .
$$

The condition imposed on $\tilde{g}$ so that it is in $\mathcal{O}$ is that the $\lambda_{p}$ so defined be a germ of a submersion at the graph of $a\left(b_{j p}\right)$. If $1 \leq j<p$ and $x \in W_{j p}^{\prime}$, then $\lambda_{p}(x)=\tilde{g}_{p j}(x) \circ b_{j p}(x)$. In fact for such an $x, \lambda_{p}(x)=\Sigma_{k<p} \phi_{k}(x) \cdot\left(\tilde{g}_{p k}(x) \circ\right.$ $\left.b_{k p}(x)\right)$. If $i \leq k<p$ and $\phi_{k}(x) \neq 0$, then $x \in W_{j k p}$ and

$$
\begin{aligned}
\tilde{g}_{p k}(x) \circ b_{k p}(x) & =\tilde{g}_{p j}(x) \circ \tilde{g}_{j k}(x) \circ b_{k p} \\
& =\tilde{g}_{p j}(x) \circ b_{j k}(x) \circ b_{k p}(x)=\tilde{g}_{p j}(x) \circ b_{j p}(x) .
\end{aligned}
$$

For $x \in W_{j p}^{\prime}$ we have then

$$
\left(1 \times \lambda_{p}\right) \circ e_{p} \circ e_{j}^{-1}=\left(1 \times \lambda_{p}\right) \circ\left(1 \times b_{p j}\right)=1 \times \tilde{g}_{p j}
$$

which completes the inductive step and the proof of the lemma.

II. Stability and instability of representations.

1. We begin investigating stability questions for $\mathfrak{F}_{X}$ via the stability of representations of $\Pi_{1}(X)$. Here stability means $C^{0}$-stability.

Up to this point we have suppressed, to a large extent, reference to the differentiability class of the foliations considered, the integer $t$. Since the choice of $t$ is relevant in this part, we make it explicit. The dictionary for this is

$$
\mathfrak{F}_{X}=\mathfrak{F}_{X}, \quad \mathcal{F}_{X}^{r}=\mathfrak{F}_{X}^{r}, \quad \text { Diff }=\text { Diff }^{t} .
$$


As before, the integer $t$ identifies the topology (the $C^{t}$-topology) used in each of these spaces as well as in the spaces Rep and $\{\text { Rep }\}^{r}$ constructed using Difft.

In the case of a closed orbit of a differential equation, the necessary and sufficient condition for stability is that the Poincaré transform be hyperbolic [Marcus]. Recall that a linear map is hyperbolic if its eigenvalues miss the unit circle and a diffeomorphism germ, $f \in \operatorname{Diff}^{t}\left(\mathbf{R}^{k}, 0\right)$ is hyperbolic iff $f^{\prime}(0)$ is a hyperbolic linear map. The following result is well known:

Proposition. If $\rho \in \operatorname{Rep}\left(Z, \operatorname{Diff}^{t}\left(\mathbf{R}^{k}, 0\right)\right)$, then $\rho$ is stable iff $\rho(1)$ is byperbolic.

Notation. If $\sigma \in \mathcal{F}_{X}$, we denote by $\rho_{\sigma}$ the corresponding class in $\left\{\operatorname{Rep}\left(Z, D_{i f f}{ }^{t}\left(\mathbf{R}^{k}, 0\right)\right)\right\}^{t}$. Since hyperbolicity of an element of $\operatorname{Diff}^{t}\left(\mathbf{R}^{k}, 0\right)$ implies the hyperbolicity of any Homeo $s\left(R^{k}, 0\right)$-conjugate for $s>0$, we can speak of the hyperbolicity of $\rho_{\sigma}(1)$, hence of $\rho_{\sigma}$. Thus we have

Theorem 2. Let $\sigma \in \mathcal{F}_{X}$ and let $\Pi_{1}(X)=Z$, then $\sigma$ is stable at $X$ iff $\rho_{\sigma}$ is byperbolic.

This theorem is an instance of the stability of a foliation or a representation depending only on the 1-jet of the representation.

We consider $\operatorname{GL}(k, \mathbf{R})$ as a subgroup of $\operatorname{Diff}^{t}\left(\mathbf{R}^{k}, 0\right)$ and let $j^{1}: \operatorname{Diff}^{t}\left(\mathbf{R}^{k}, 0\right)$ $\rightarrow \mathrm{GL}(k, R)$ be the 1-jet at zero map. We have the commutative diagram:

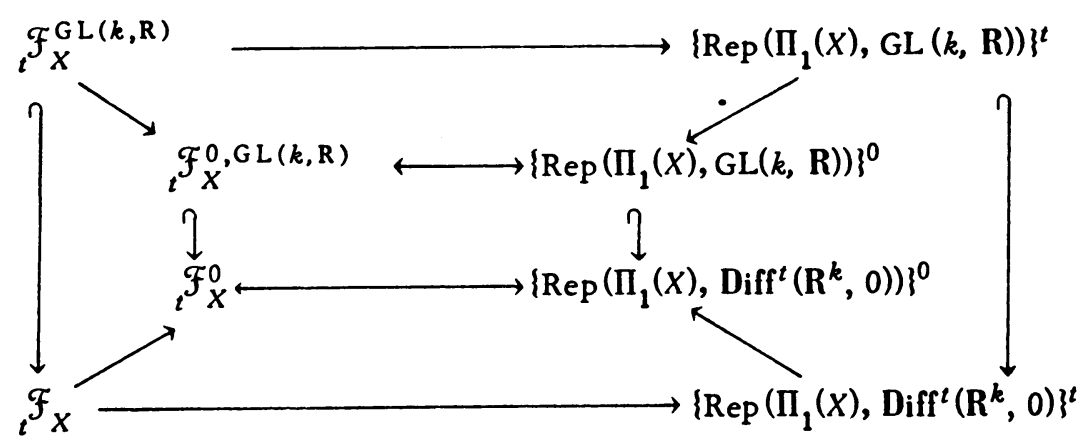

where all the horizontal maps are homeomorphisms (given by the corollary Theorem 1 of $\$ 2)$. The vertical maps are those induced by the inclusion of $\operatorname{GL}(k, \mathbf{R})$ in $\operatorname{Diff}\left(\mathbf{R}^{k}, 0\right)$, and all the diagonal maps are forgetful.

Definition. An element $\rho \in \operatorname{Rep}\left(\Pi_{1}(X), \mathrm{GL}(k, \mathbf{R})\right)$ is linearly stable if the projection $\operatorname{Rep}\left(\Pi_{1}(X), \operatorname{GL}(k, \mathbf{R})\right) \rightarrow\left\{\operatorname{Rep}\left(\Pi_{1}(X), \mathrm{GL}(k, \mathbf{R})\right)\right\}^{0}$ is constant on a neighborhood of $\rho$.

Using this definition we can speak of linearly stable, linear foliations where a linear foliation at $X$ is one whose class is in ${ }_{t} \mathcal{F}_{X}^{\mathrm{GL}(k, R)}$. 
Question. What is the relationsbip between the stability of a foliation at $X$ and the linear stability of its 1-jet, linear foliation?

In other words, if $\rho \in \operatorname{Rep}\left(\Pi_{1}(X), \operatorname{Diff}^{t}\left(\mathbf{R}^{k}, 0\right)\right)$ how does the stability of $\rho$ relate to the linear stability of $j^{1} \circ \rho$ ?

In the case $t=1$, it is trivial that if $\sigma \in{ }_{1} F_{X}$ is stable then so is its 1-jet linear foliation. This, of course, is a consequence of the fact that a neighborhood of $\rho_{\bar{\sigma}} \in\left\{\operatorname{Rep}\left(\Pi_{1}(X), \operatorname{Diff}^{1}\left(\mathbf{R}^{k}, 0\right)\right\}^{1}\right.$ contains a neighborhood in $\left\{\operatorname{Rep}\left(\Pi_{1}(X), \operatorname{GL}(k, R)\right\}^{1}\right.$

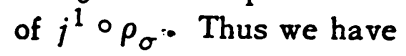

Proposition. If there are no stable elements in $\operatorname{Rep}\left(\Pi_{1}(X), \operatorname{GL}(k, \mathbf{R})\right)$, then there are no stable elements in ${ }_{1} \mathcal{F}_{X}$.

So far we know that if $\Pi_{1}(X)$ is finite, any $\rho \in \operatorname{Rep}\left(\Pi_{1}(X), \operatorname{Diff}^{t}\left(\mathbf{R}^{k}, 0\right)\right)$ is stable. If $\Pi_{1}(X)=Z$, then $\rho \in \operatorname{Rep}\left(Z, \operatorname{Diff}^{t}\left(\mathbf{R}^{k}, 0\right)\right)$ is stable iff $j^{1} \circ \rho(1)$ is hyperbolic. In the next section we show that there are no stable representations of $Z^{n}$ in $G L(k, \mathbf{R})$ for $n>1$. The next question would be the stability of finitedimensional linear representations of simple or semisimple groups. Is it true that all finite dimensional linear representations of a finitely presented simple group are stable? (This would be analogous to the situation for Lie groups.) We have been informed that Stallings has produced a finitely presented infinite simple group. Thus the question is not a priori subsumed under the finite $\Pi_{1}(X)$ case.

2. We now consider representations of $Z^{n}$ into $\mathrm{GL}(k, \mathbf{R})$ and show that there are no stable ones. The proof uses a number of elementary algebra arguments which are included since we could find no reference for them.

Lemma. Let $A, B \in \mathrm{GL}(k, \mathbf{R})$; then if $A$ bas an eigenvalue of absolute value one and $B$ does not then $A$ and $B$ are not conjugate by a bomeomorphism.

Proof. If $A$ and $B$ were conjugate by a homeomorphism the absolute-valueone-eigenvector of $A$ would be recurrent for $B$. But $B$ has only 0 as recurrent point.

Definition. Let $G$ be any group. A representation $\rho \in \operatorname{Rep}(G, \operatorname{GL}(k, \mathbf{R}))$ is byperbolic if the eigenvalues of $\rho(x)$ are all off the unit circle for $x \neq$ idenitiy in $G$.

Proposition. For $n>1$, the set of byperbolic representations and its comple. ment are dense sets in $\operatorname{Rep}\left(Z^{n}, \mathrm{GL}(k, \mathbf{R})\right)$.

This proposition implies

Theorem 3. Let $n>1$, then there are no stable elements in $\operatorname{Rep}\left(Z^{n}, G L(k, R)\right)$.

Corollary. Let $X$ be a compact manifold with $\Pi_{1}(X)=Z^{n}$ for $n>1$. Then there are no stable elements in ${ }_{1} \mathcal{F}_{X}$. 


\section{Proof of the proposition.}

Lemma. Let $G$ be an abelian group and $\rho \in \operatorname{Rep}(G, G L(k, \mathbf{R}))$. Then $\mathbf{R}^{k}=$ $E_{1} \oplus \ldots \oplus E_{n}$ where $E_{i}$ is an invariant subspace for the representation and in complex form $\rho(g) \mid E_{i}=\gamma_{i}(g) I+N_{i}(g)$ where $\gamma_{i}(g) \in C-\{0\}$ and $N_{i}(g)$ is nilpotent and $\gamma_{i}$ is a bomomorphism of $G$ into the multiplicative group of nonzero complex numbers.

Proof. Let $g \in G$ and let $\lambda$ be an eigenvalue of $\rho(g)$. For sufficiently high $m, \operatorname{ker}(\lambda I-\rho(g))^{m}=\operatorname{ker}(\lambda I-\rho(g))^{m+1}$. Further this kernel is invariant under all linear maps which commute with $\rho(g)$. On this subspace $\rho(g)=\lambda I+(\rho(g)-\lambda I)$. Using the finite dimensionality of $\mathbf{R}^{k}$, we get the direct sum decomposition. That the $\gamma_{i}$ are elements of $\operatorname{Rep}\left(G, C^{*}\right)$ is obvious.

To complete the proof of the proposition, we take any representation $\rho$, and restrict to one of the subspaces $E_{i}$ of the lemma. Let $e_{1}, \cdots, e_{n}$ be a set of generators of $Z^{n}$. We can change the representation by multiplying the $\rho\left(e_{j}\right)$ by a real number $r_{j} \neq 0$. The representation $g \rightarrow \gamma_{i}(g)$ will hit the unit circle iff there are integers $m_{j}$ such that $\left|\operatorname{det}\left(\Pi_{j} \rho\left(e_{j}\right)^{m}{ }^{j}\right)\right|=1$.

This is equivalent to the existence of integers $m_{j}$ such that

$$
\sum_{j} m_{j} \ln \left|\gamma_{i}\left(e_{j}\right)\right|=0
$$

The set of points which satisfy $\sum_{j=1}^{n} m_{j} x_{j}=0$, for $\left(m_{1}, \cdots, m_{n}\right) \in Z^{n}$ is a countable union of hyperplanes in $\mathbf{R}^{n}$ which contains the set of rational points. Thus this set and its complement is dense in $\mathbf{R}^{n}$. By multiplying the $\rho\left(e_{j}\right)$ by $r_{j}$ arbitrarily close to 1 we may move the $n$-tuple $\left(\ln \left|\gamma_{i}\left(e_{1}\right)\right|, \ldots, \ln \left|\gamma_{i}\left(e_{n}\right)\right|\right)$ into either of these sets.

The proof given here is valid if $Z^{n}$ is replaced by any group of the form $Z^{n} \times G$, for $G$ arbitrary, and $n>1$. Thus we have actually proven

Theorem. Let $n>1$, and let $G$ be an arbitrary group. Then there are no stable elements in $\operatorname{Rep}\left(Z^{n} \times G, G L(k, R)\right)$.

Corollary. Let $X$ be a compact manifold with $\Pi_{1}(X)$ of the form $Z^{n} \times G$ for $n>1$. Then there are no stable elements in ${ }_{1} \mathcal{F}_{X}$.

Question. Are there any stable representations of (finitely presented) solvable group into $\mathrm{GL}(k, \mathbf{R})$ ?

We expect the answer to this question to be "essentially no", and for that reason we raised the question of stability of linear representations only for simple and semisimple groups. 


\section{REFERENCES}

[H $\mathrm{H}_{1}$ ]. A. Haefliger, Structures feuilletees et cohomologie à valeur dans un faisceau de groupoides, Comment. Math. Helv. 32 (1958), 248-329.

[ $\left.\mathrm{H}_{2}\right]$. - Variétés feuilletées, Ann. Scuola Norm. Sup. Pisa (3) 16 (1962), 367397. MR 32 \#6487.

$\left[\mathrm{H}_{3}\right]$.

Feuilletages sur les variétés ouvertes, Topology 9 (1970), 183-194. MR 41 \#7709.

[Hirsch 1]. M. W. Hirsch, Foliations and non-compact transformation groups, Bull. Amer. Math. Soc. 76 (1970), 1020-1023. MR $45 \# 1189$.

[Hirsch ${ }_{2}$ ]. - - Stability of compact leaves of foliations, Proc. Internat. Conf. on Dynamical Systems, Salvador, Brasil 1971 (to appear).

[H.P.S.]. M. W. Hirsch, C. C. Pugh and M. Shub, Invariant manifolds, Bull. Amer. Math. Soc. 76 (1970), 1015-1019. MR $45 \# 1188$.

[Marcus]. L. Marcus, Structurally stable differentiable systems, Ann. of Math. (2) 73 (1961), 1-19.

[M]. J. Milnor, Microbundles. I, Topology 3 (1964), suppl. 1, 53-80. MR 28 \#4553b.

[N]. Z. Nitecki, Differentiable dynamics, M.I.T. Press, Cambridge, Mass., 1972.

[R]. G. Reeb, Sur certaines propriétés topologiques des variétés feuilletées, Actualités Sci. Indust., no. 1183 = Publ. Inst. Math. Univ. Strásbourg 11, Hermann, Paris, 1952, pp. 91-154, 157-158. MR 14, 1113.

[St]. N. Steenrod, The topology of fibre bundles, Princeton Math. Series, vol. 14, Princeton Univ. Press, Princeton, N. J., 1951. MR 12, 522.

DEPARTMENT OF MATHEMATICS, BRANDEIS UNIVERSITY, WALTHAM, MASSACHUSETTS 02154 (Current address of $\mathrm{H}$. I. Levine)

Current address (Michael Shub): Department of Mathematics, Queens College, Flushing, New York 11367 